\title{
Signal transducer and activator of transcription gene polymorphism in type 1autoimmune hepatitis
}

\author{
Nazih M.Alnady ${ }^{1}$, Ola G. Behairy ${ }^{1}$, Shuzan A.Mohammed ${ }^{2}$ and Walaa A.Ibrahim ${ }^{3}$
}

${ }^{1}$ Pediatrics department, Faculty of Medicine, Benha University, Egypt

${ }^{2}$ Medical Biochemistry department, Faculty of Medicine, Benha University, Egypt

${ }^{3}$ M.B.B.Ch, Faculty of Medicine, Benha University, Egypt

Email:

\begin{abstract}
Background: Autoimmune hepatitis (AIH) is one of the chronic inflammation types of the liver, characterized by hypergammaglobulinemia and autoantibodies formation. Signal transducer and activator of transcription 4 (STAT4) gene play an important role in dendritic cells, macrophages and activated peripheral blood monocytes. Many previous studies reported the association between STAT4 and some autoimmune diseases, and in a mouse model confirmed the association, STAT4 is an important factor in Th1-dependent liver injury. Aim: to assess the association of a signal transducer and activator of transcription STAT4 gene polymorphism and their interactions with type1 autoimmune hepatitis in children methods: This is across-sectional study, it was performed on 50 patients ages with autoimmune hepatitis from the Pediatric Hepatology Clinic of Benha University Hospitals. All children were subjected to full history taking, physical examination, anthropometric measurements, abdominal examination, serum transaminases, serum bilirubin and serum albumin, PT, PTT, INR, antinuclear antibodies (ANA), anti-smooth muscle antibody (ASMA), antimitochondrial antibody (AMA), anti-liver kidney microsomal antibody (Anti LKM), total IgG levels, hepatitis markers, liver biopsy and genotyping for STAT4 gene.Genotyping of STAT4 gene polymorphism. Conclusion: rs7574865 and rs7582694 in STAT4 gene minor alleles are associated with increased type 1 AIH risk.
\end{abstract}

Keywords: Autoimmune, Hepatitis, Signal transducer and activator of transcription 4.

\section{Introduction}

Autoimmune hepatitis (AIH) is a chronic liver disease affect the immunological tolerance to autologous liver tissue. This immune system imbalance typically causes inflammation of hepatocytes with increase the levels of circulating autoantibodies, hypergammaglobulinemia and fluctuating increases in serum transaminases and immunoglobulin G levels. [1]

Autoimmune hepatitis is usually diagnosed after other causes of chronic hepatitis as viral, metabolic or drug-induced causes have been ruled out. Although it is uncommon in children, autoimmune hepatitis may manifest itself with severe hepatocellular damage and early fibrosis. Early diagnosis and treatment will improve the morbidity and decrease the mortality. In other cases, the only final rescue for these children is liver transplantation. [2]

Autoimmune hepatitis has been classified into two types based on the serum autoantibody levels. Type $1 \mathrm{AIH}$ is characterized by presence of antinuclear antibody (ANA), anti-smooth muscle antibody (ASMA) or both. Type 2 AIH is characterized by presence of anti-liver kidney microsomal (LKM) and/or anti-liver cytosol (LC1) antibodies. [3]

The etiology of AIH is unknown, but is thought to have both a genetic and an environmental basis. In the genetic base there are confirmed susceptibility genes as HLA DRB1 gene and nonconfirmed ones as Non-HLA susceptibility genes.[4]

With the emergence of genome-wide association studies (GWAS), there has been a dramatic increase in genetic discoveries for many complex genetic autoimmune diseases, such as type 1 diabetes and rheumatoid arthritis (RA).[5]

Investigating the genetic polymorphism of a signal transducer and activator of transcription STAT4 with autoimmune diseases, many recent studies suggested a significant association of STAT4 gene polymorphism with systemic lupus erythematosus (SLE), rheumatoid arthritis (RA), Crohn's, Sjögren's disease (SD), systemic sclerosis, Behcet's disease, and type-1 diabetes (T1D) as well as psoriasis, thus indicating common genetic involvement in multiple autoimmune diseases.[6]

Signal transducer and activator of transcription 4 (STAT4) is a transcription factor involved in the signaling pathways of several cytokines, playing an essential role in the development of inflammation in various immune-mediated diseases. The expression of (STAT4) gene occurs in activated peripheral blood monocytes, dendritic cells and macrophages causing inflammation in humans.[7]

Interleukin (IL)-12 activates it, causing $\mathrm{T}$ helper (Th) 1 and Th 17 differentiation, monocyte activation, and the release of interferon (IFN)-a. STAT4 may play a critical role in the development of autoimmune diseases since Th1 and Th17 cells have the ability to trigger autoimmunity. [8]

The aim of this study was to see if there was a connection between a signal transducer and a transcriptional activator STAT4 gene polymorphism and their interactions with type 1 autoimmune hepatitis in children. [9]

\section{Patients and methods}

This cross-sectional study was done in cooperation between the Pediatrics Department and the Molecular Biology Unit, Faculty of Medicine, Benha University on 50 patients with autoimmune hepatitis diagnosed according to the International Autoimmune Hepatitis Group (IAIHG) revised scoring system and American Association for the Study of Liver Diseases 
(AASLD) guidelines [10] from the Pediatric Hepatology Clinic of Benha University Hospitals and National liver institute, Menofia University

Written informed consent was obtained from the parents or health care providers of each child. Approval of the study was obtained from the Ethical Committee of Scientific Research, Faculty of Medicine, Benha University.

All children with other chronic liver diseases, viral hepatitis, drug induced hepatitis, fatty liver disease and metabolic disorders were excluded from the study.

All children were subjected to Full history taking, physical examination, anthropometric measurements: including weight, height and Z-score were recorded. body mass index (BMI) was calculated as $\mathrm{kg} / \mathrm{m}^{2}$, abdominal examination, serum transaminases, serum bilirubin and serum albumin, prothrombin time (PT), prtial thrombin time (PTT), international normalized ratio (INR), antinuclear antibodies (ANA), anti-smooth muscle antibody (ASMA), antimitochondrial antibody (AMA), anti-liver kidney microsomal antibody (Anti LKM), total immunoglobulin ( $\mathrm{IgG}$ ) levels, hepatitis markers, liver biopsy and genotyping for STAT4 gene.

\subsection{Genotyping of STAT4 gene polymorphism}

A venous blood sample $(3 \mathrm{ml})$ was taken from each subject. Two ml were put on EDTA and kept at $200 \mathrm{C}$ for genotyping. All the other laboratory investigations were obtained from the patient records. One SNP (rs7574865) was detected by RFLP and the 2nd SNP (rs7582694) was detected by real-time PCR. DNA was extracted from $200 \mu$ l blood sample; using Purelink ${ }^{\circledR}$ Genomic DNA minikit Catalog No. K182001 (Invitrogen, Life Technologies) according to manufacturer's instructions. A digestion was performed to degrade RNA in the sample and minimize RNA contamination) also digestion by proteinase $\mathrm{K}$ was done for efficient lysis of cells. Elution of DNA was done by 50 elution buffers. The extracted DNA concentration was measured by Nanodrop Spectrophotometer 2000 (Thermo-Fisher Scientific, Wilmington, USA). Readings were taken at wave lengths 260 and $280 \mathrm{~nm}$. according to that reported by Wilfinger et al., (1997).[11] The ratio of optical density (OD) at $260 \mathrm{~nm}$ and $280 \mathrm{~nm}$ provides an estimate of DNA purity. Pure preparations of DNA have OD260/OD280 of 1.7 - 2.0. If contaminated with protein or phenol, the ratio is $<1.7$, but if contaminated with RNA, the ratio is $>2.0$. The extracted DNA concentration ranged from 20-30 ng DNA/ $\mu$ l. Genomic DNA amplification was done in $25 \mu \mathrm{l}$ reaction / a sample using primers for STAT4 rs7574865 previously reported by El-Saadany et al. (2016).[12] Amplification was done in Veriti ${ }^{\mathrm{TM}}$ Thermal Cycler (Applied Biosystems). The PCR conditions were $5 \mathrm{~min}$ at $95 \mathrm{oC}$ initial denaturation, 35 cycles (denaturation at $950 \mathrm{C}$ for $30 \mathrm{~s}$, annealing at $56 \mathrm{oC}$ for $30 \mathrm{~s}$, extension at $72 \mathrm{oC}$ for $1 \mathrm{~min}$ ) and then final extension at $720 \mathrm{C}$ for $5 \mathrm{~min}$. PCR products $(10 \mu \mathrm{l})$ and 100 base pair ladder $(5 \mu \mathrm{L})$ were resolved in $2.5 \%$ agarose gel stained with $0.3 \mathrm{ug} / \mathrm{ml}$ ethidium bromide to check the PCR products at $147 \mathrm{bp}$ fragment.
RFLP was done for STAT4 (rs7574865) by Fastdigest HpaI restriction enzyme (Thermo Fisher Scientific) in $30 \mu$ l total volume by mixing: $10 \mu$ of PCR products $+1 \mu \mathrm{l} \mathrm{HpaI}$ restriction enzyme ( 1 unit) + $2 \mu \mathrm{l} 10 \mathrm{X}$ buffer $+17 \mu \mathrm{l}$ nuclease-free water. The digestion mixtures were incubated at $37{ }^{\circ} \mathrm{C}$ for 2 hours then inactivated by incubation at $65^{\circ} \mathrm{C}$ for $10 \mathrm{miDNA}$ fragments $(10 \mu \mathrm{L})$ and 100 bp ladder $(5 \mu \mathrm{L})$ were separated on $2 \%$ agarose gel stained with $0.3 \mathrm{ug} / \mathrm{ml}$ ethidium bromide. The bands (pre- and post-digestion) were visualized using UV transilluminator (254 nm) and imaged with a digital camera 8 mega pixel.

The image was analyzed by computer software (Alpha InoTech Gel Documentation System). Predigestion bands were visualized at $147 \mathrm{bp}$. Postdigestion; the $\mathrm{T}$ allele gave two fragments (122 bp and $25 \mathrm{bp}$ ), while the $\mathrm{G}$ allele was uncut (147 bp). The small band ( $25 \mathrm{bp})$ was lost in the gel. The success rate was $95 \%$. The failed PCR were rerun by the same conditions.

Allele discrimination of STAT4 (rs7582694) SNP by PCR by using TaqMan SNP Genotyping assay (assay on demand, Applied Biosystem, Foster City, California, USA) through a Stepone Real Time PCR System (Applied Biosystem, Foster City, USA) according to the manufacturer's instructions. The Taqman assay was supplied as $40 \mathrm{X}$ concentration. The assay contained sequence specific primers for both alleles (major $\mathrm{G}$ and minor $\mathrm{C}$ ) and 2 Taqman probes; one probe labeled with VIC dye detects the $\mathrm{G}$ allele and the other labeled with FAM detects the $\mathrm{C}$ allele. Each 40X SNP genotyping assay was diluted before real time PCR mix was done to $20 \mathrm{X}$ working stock with $1 \mathrm{X}$ TE buffer. Then it was aliquoted and stored at -20 oC.

\subsection{Statistical analysis}

The collected data were tabulated and analyzed using SPSS version 16 software (SpssInc, Chicago, ILL Company). Genotype distributions in the studied groups were in Hardy-Weinberg equilibrium for gene polymorphisms (data not shown). Hardy-Weinberg equilibrium was calculated according to OEGE Online Encyclopedia for Genetic Epidemiology studies.[13] Categorical data were presented as number and percentages while quantitative data were expressed as mean \pm standard deviation, median and range. Chi square (X2), Fisher's exact test (FET) and Kappa test were used to analyze categorical variables. Continuous data were tested for normality using Shapiro-Wilks test assuming normality at $\mathrm{P}>0.05$, using student " $\mathrm{t}$ " test to assess difference among 2 independent groups regarding normally distributed variables or Man Whitney $U$ test for non-parametric ones. Binary logistic regression analysis was run to detect the predictor allele of AIH. $\mathrm{P} \leq 0.05$ was considered significant(S), $\mathrm{P}$ value $>0.05$ is non-significant (NS) \& $\mathrm{P} \leq 0.001$ is highly significant (HS).

\section{Results}

Fourteen patients had eczema; 6 patients had inflammatory bowel disease (IBD). 11 patients with their siblings have autoimmune disease, 5 patients have 
father and other siblings with autoimmune disease, and 5 patients with their mothers have autoimmune disease. There was statistically significant difference between studied group regarding autoimmune disease and autoimmune disease in first degree relative which was higher in autoimmune hepatitis group. However, there was no statistically difference between studied group regarding sex, age and Consanguinity Table (1).

The majority of patients suffered from jaundice (72.8\%), abdominal distention (35.2\%) and fatigue (33.6\%) abdominal pain (19.2\%). That most of patients had no ascites and only $12 \%$ had mild ascites.35.2 \% of patients with hepatosplenomegaly and $64.8 \%$ had hepatomegaly only Fig. (1).

Table (2) shows that there was statistically significant difference between studied group regarding the weight on centile, body mass index (BMI) on centile and the height on centile as all were low in Auto immune hepatitis group However, there was no statistically difference between studied group regarding the head circumference.

Table (1) Socio-demographic characters of the studied groups.

\begin{tabular}{|c|c|c|c|c|c|c|c|}
\hline \multirow[t]{2}{*}{ Variable } & & \multicolumn{2}{|c|}{$\begin{array}{c}\text { Auto immune } \\
\text { hepatitis group } \\
(\mathrm{N}=125)\end{array}$} & \multicolumn{2}{|c|}{$\begin{array}{l}\text { Control group } \\
\qquad(\mathrm{N}=125)\end{array}$} & \multirow[t]{2}{*}{ Test of sig } & \multirow[t]{2}{*}{$\mathbf{P}$} \\
\hline & & No. & $\%$ & No. & $\%$ & & \\
\hline \multirow{2}{*}{ Sex } & Male & 57 & $(45.6)$ & 60 & $(48.0)$ & \multirow{2}{*}{$X^{2}=0.15$} & \multirow{2}{*}{$0.70(\mathrm{NS})$} \\
\hline & Female & 68 & $(54.4)$ & 65 & $(52.0)$ & & \\
\hline Age (years) & $\begin{array}{l}\text { Mean } \pm \text { SD } \\
\text { Range }\end{array}$ & \multicolumn{2}{|c|}{$\begin{array}{c}9.9 \pm 3.7 \\
4-16\end{array}$} & \multicolumn{2}{|c|}{$\begin{array}{c}9.3 \pm 4.3 \\
2-16\end{array}$} & $\mathrm{ZMWU}=1.05$ & $0.29(\mathrm{NS})$ \\
\hline \multirow{2}{*}{ Consanguinity } & Positive & 26 & (20.8) & 17 & (13.6) & \multirow{2}{*}{$X^{2}=2.27$} & \multirow[b]{2}{*}{$0.13(\mathrm{NS}$} \\
\hline & Negative & 99 & $(79.2)$ & 108 & (86.4) & & \\
\hline \multirow{5}{*}{$\begin{array}{l}\text { Other Autoimmune } \\
\text { Disease } \\
\text { Autoimmune Disease } \\
\text { in first degree } \\
\text { relative }\end{array}$} & No & 105 & $(84.0)$ & 122 & (97.6) & \multirow{2}{*}{$X^{2}=13.8$} & \multirow{2}{*}{$\begin{array}{c}<0.001 \\
(\mathrm{HS})\end{array}$} \\
\hline & Yes & 20 & $(16.0)$ & 3 & $(2.4)$ & & \\
\hline & No & 104 & $(83.2)$ & 125 & $(100.0)$ & \multirow{3}{*}{$X^{2}=22.9$} & \multirow{2}{*}{$\begin{array}{c}<0.001 \\
(\mathrm{HS})\end{array}$} \\
\hline & Yes & 21 & $(16.8)$ & 0 & $(0.0)$ & & \\
\hline & & & & & & & $\mathrm{X}^{2}$ \\
\hline
\end{tabular}

$\mathbf{Z}_{\mathbf{M W U}} \rightarrow \mathrm{Z}$ value of Mann Whitney U test. $\mathrm{X}^{2}=$ Chi square test. SD: stander deviation. NS: non-significant. HS: highly significant. S: significant

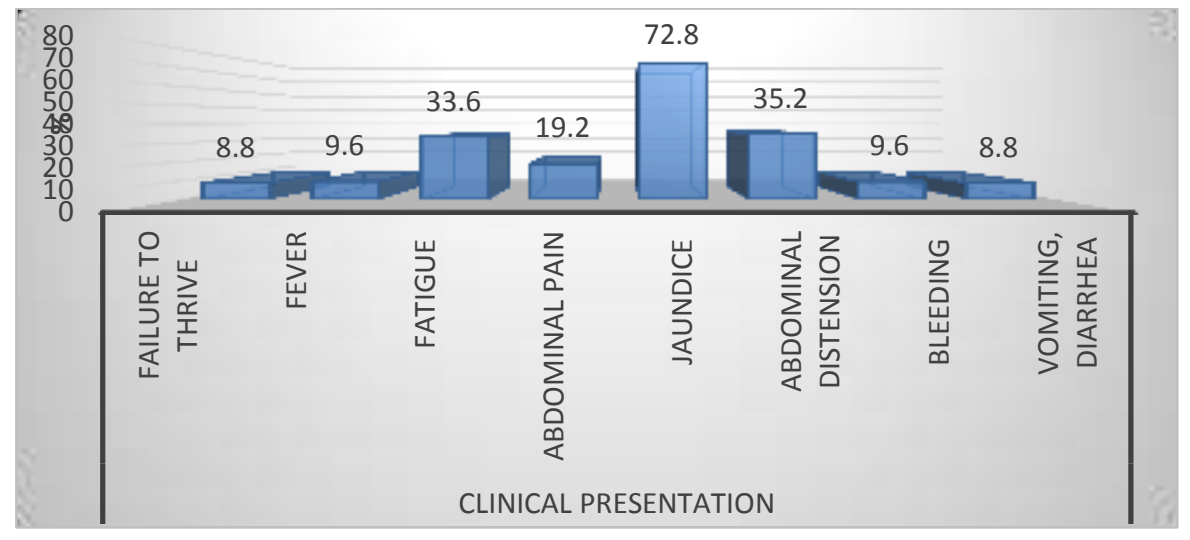

Fig. (1) Clinical presentation of the studied patients

Table (2) Anthropometric measurements among the studied groups

\begin{tabular}{|c|c|c|c|c|c|c|c|c|c|c|}
\hline \multirow[t]{2}{*}{ Variable } & \multicolumn{4}{|c|}{$\begin{array}{l}\text { Auto immune hepatitis group } \\
\qquad(n=125)\end{array}$} & \multicolumn{4}{|c|}{$\begin{array}{l}\text { Control group } \\
(\mathrm{N}=125)\end{array}$} & \multirow{2}{*}{$\begin{array}{c}\text { St."t" } \\
\text { test }\end{array}$} & \multirow[t]{2}{*}{$\mathbf{P}$} \\
\hline & Mean & \pm & SD & Range & Mean & \pm & SD & Range & & \\
\hline Weight (kg) & 27.8 & \pm & 10.5 & $15-50$ & 32.4 & \pm & 15.7 & $15-66$ & 2.71 & $0.007(\mathrm{~S})$ \\
\hline Weight centile & 15.5 & \pm & 10.4 & $5-55$ & 32.0 & \pm & 15.8 & $9-88$ & $9.67 *$ & $<0.001(\mathrm{HS})$ \\
\hline Height (cm) & 126.0 & \pm & 21.4 & $81-165$ & 134.5 & \pm & 25.0 & $91-178$ & 2.24 & $0.004(\mathrm{~S})$ \\
\hline Height centile & 20.7 & \pm & 13.9 & $8-75$ & 36.2 & \pm & 12.0 & $21-78$ & $8.2^{*}$ & $<0.001(\mathrm{HS})$ \\
\hline $\mathrm{HC}(\mathrm{cm})$ & 50.4 & \pm & 2.16 & $48-55$ & 50.9 & \pm & 2.6 & $47-55$ & 1.76 & $0.08(\mathrm{NS})$ \\
\hline HC centile & 46.0 & \pm & 19.7 & $14-72$ & 49.5 & \pm & 19.5 & $18-75$ & 1.64 & $0.1(\mathrm{NS})$ \\
\hline BMI (kg/m²) & 15.8 & \pm & 1.97 & $12-23$ & 16.7 & \pm & 2.83 & $12.4-23.9$ & 3.07 & $0.002(\mathrm{~S})$ \\
\hline BMI centile & 23.0 & \pm & 18.1 & $5-79$ & 44.1 & \pm & 20.1 & $6-88$ & $8.0 *$ & $<0.001(\mathrm{HS})$ \\
\hline
\end{tabular}

*Denote significant value of Mann Whitney U test. BMI: body mass index. HC: head circumference. Cm: 
centimeter. Kg: kilogram. NS: non-significant. HS: highly significant. S: significant

There was statistically significant difference between studied group regarding alanine aminotransferase (ALT), aspartate aminotransferase (AST), alkaline phosphatase (ALP), serum bilirubin (total, direct), immunoglobulin $\mathrm{G}$ and the bleeding profile (PT, PTT, INR) as all were higher in autoimmune hepatitis group, while serum albumin and proteins were lower in autoimmune hepatitis group. However, there was no significant difference between both groups regarding serum creatinine (Figure 2\&3). $80 \%$ of the patients had positive autoantibodies (ANA, ASMA), no patients had positive AMA or Anti LKM. According to original scoring system for diagnosis of autoimmune hepatitis (RODC) 63.2\% of patients were definitely diagnosed as autoimmune hepatitis while $36.8 \%$ were probably diagnosed. According to SDC (simplified scoring system) $80 \%$ of patients had definite diagnosis of $\mathrm{AIH}$, while $20 \%$ only had probable diagnosis. About 45. $6 \%$ of patients were treated by steroids and $54.4 \%$ were treated by steroids azithoprine. $63.2 \%$ of patients showed complete recovery with treatment while $36.8 \%$ showed relapse of the disease.

$80.8 \%$ of the study group had piecemeal interface hepatitis and the other $20 \%$ vary between rosette and rosette ballooning (14.8\% rosette and $4.8 \%$ rosette ballooning) and the type of the cells vary between plasma cells mostly (69.6\%), lymphocytes (45.6), Esinophils (16.8\%) and mononuclear cells (16\%) in order, the studied group also showed that most of patients were in early fibrosis stage $(48.8 \%$ fibrosis stage 1,2 and $32 \%$ fibrosis stage 3 and $19.2 \%$ fibrosis stage 4). According to the histological activity index $62.4 \%$ of patients with mild activity while $28 \%$ of patients with moderate activity and $9.6 \%$ of patients only have sever activity index. According to the Child - Pugh classification most of the study group were class B (76.8\%) and $12.8 \%$ class A and only $10 \%$ class C.

Fig. (2) and (3) showed that, individuals with GT genotype were two folds increased risk of $\mathrm{AIH}$ development than GG individuals (30.4\% vs. $21.6 \%$ ). Also, those with TT genotype were about four folds increased risk of $\mathrm{AIH}$ development than GG ones $(20.8 \%$ vs. $8.0 \%)$. All these associations were statistically significant $(\mathrm{P}=0.019$ and $=0.001$ respectively). Individuals with $\mathrm{T}$ allele were about 2.5 folds increased risk of $\mathrm{AIH}$ development than those with $\mathrm{G}$ allele (36\% vs. $18.8 \%)$ these association was statistically significant $(\mathrm{P}<0.001) . \mathrm{G}$ allele is protective but $\mathrm{T}$ allele is independent risk factor for AIH. When analyzing the dominant model of inheritance of the minor allele $\mathrm{T}$, it was found that GT+TT genotypes were significantly associated with $\mathrm{AIH}$ patients than healthy control (51.2\% vs. $29.6 \%$ ) with about two and half-fold increased risk of AIH development (P 0.001, OR 2.49). When analyzing the recessive model of inheritance of the minor allele $\mathrm{T}$, it was found that TT genotype was significantly associated with $\mathrm{AIH}$ patients than healthy control $(20.8 \%$ vs. $8.0 \%)$ with three-fold increased risk of AIH development (P 0.004, OR 3.0).

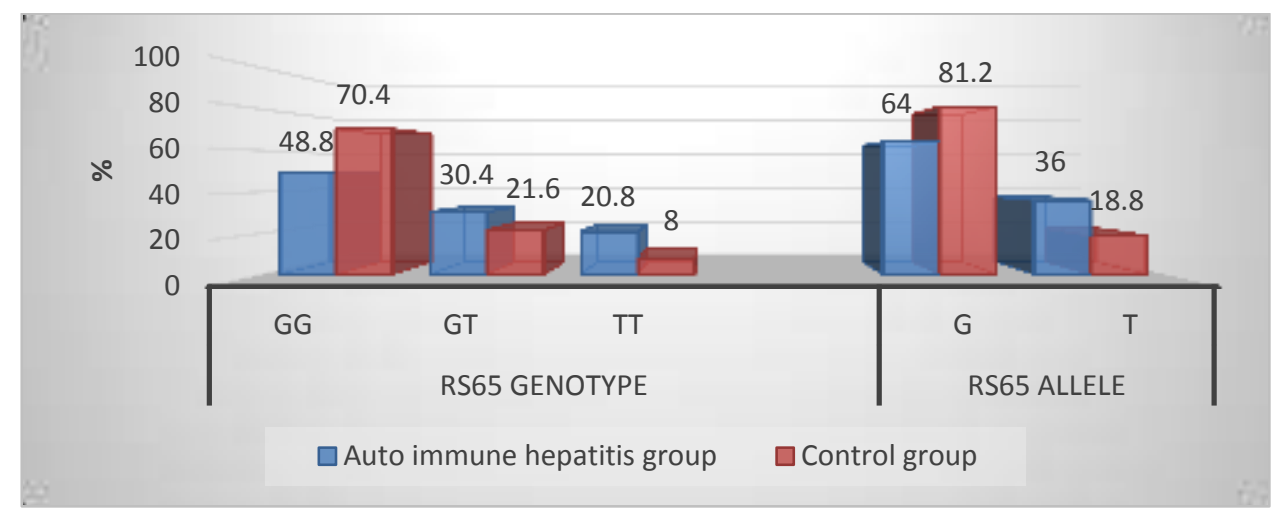

Fig. (2) Distribution of STAT 4 (rs7574865) genotype and alleles in the studied group.

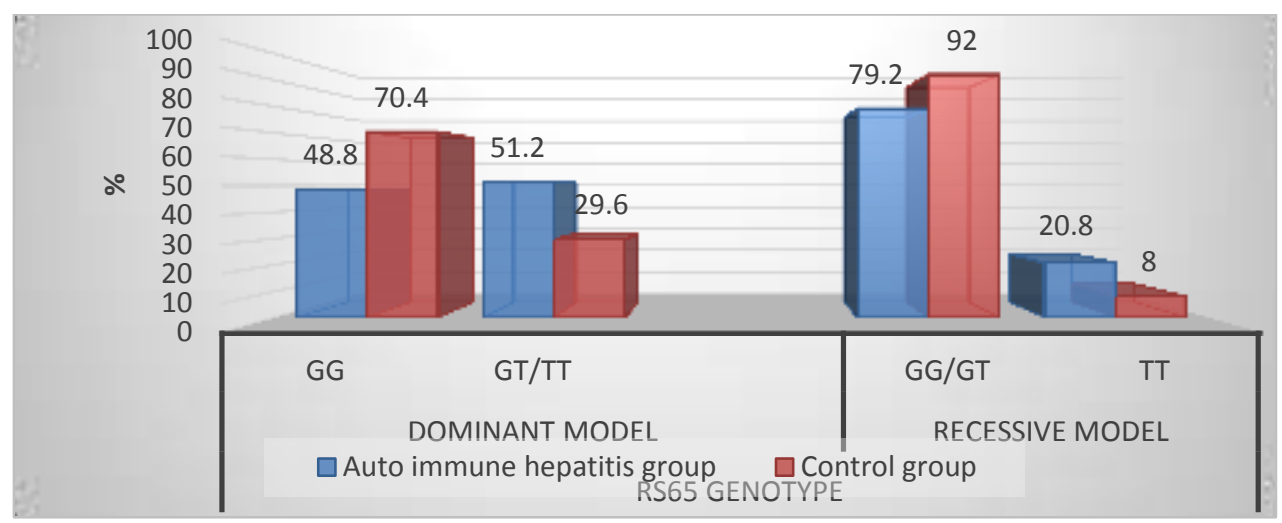

Fig. (3) Distribution of STAT 4 (rs7574865) dominant and recessive models among the studied group. 
Fig. (4) and (5) showed that individuals with GC genotype were 1.76 folds increased risk of $\mathrm{AIH}$ development than GG individuals (41.6\% vs. $32 \%$ ). Also, those with CC genotype were 3.8 folds increased risk of AIH development than GG ones (11.2\% vs. $4 \%)$. All these associations were statistically significant ( $\mathrm{P}=0.037$ and $=0.015$ respectively). Individuals with $\mathrm{C}$ allele were about 1.88 folds increased risk of $\mathrm{AIH}$ development than those with $G$ allele (32\% vs. 20\%) these association was statistically significant $(\mathrm{P}=0.002)$. $\mathrm{G}$ allele is protective but $\mathrm{C}$ allele is independent risk factor for AIH. When analyzing the dominant model of inheritance of the minor allele $\mathrm{C}$, it was found that $\mathrm{GC}+\mathrm{CC}$ genotypes were significantly associated with AIH patients than healthy control (52.8\% vs. $36 \%$ ) with 1.99 -fold increased risk of AIH development (P 0.008, OR 1.99). When analyzing the recessive model of inheritance of the minor allele $\mathrm{C}$, it was found that $\mathrm{CC}$ genotype was significantly associated with AIH patients than healthy control
(11.2\% vs. $4 \%$ ) with 3.03-fold increased risk of $\mathrm{AIH}$ development $(\mathrm{P}=0.032$, OR 3.3).

Regarding STAT4 (rs7574865) both dominant and recessive models are significant predictors of autoimmune hepatitis (dominant model was 5.63 times more risk and the recessive model was 2.77 times more risk). Regarding STAT4 (rs7582694) only the dominant model was significant predictor for autoimmune hepatitis (3.1 times more than normal) while the recessive model was not significant.

Table (3) showed that there was statistically nonsignificant relation between STAT4 (rs7574865) gene and age, sex, parents' consanguinity, other autoimmune disease and autoimmune disease in first degree relatives among the studied group.

Table (3) showed that there was statistically nonsignificant relation between STAT4 (rs7574865) gene and age, sex, parents' consanguinity, other autoimmune disease and autoimmune disease in first degree relatives among the studied group.

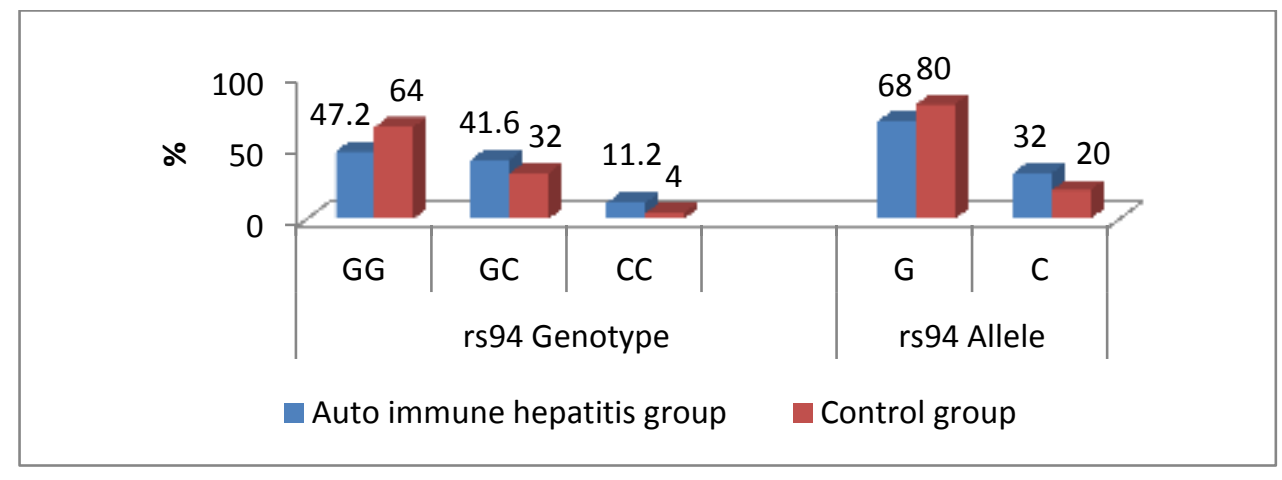

Fig. (4) Distribution of STAT 4 (RS7582694) genotype and alleles in the studied group

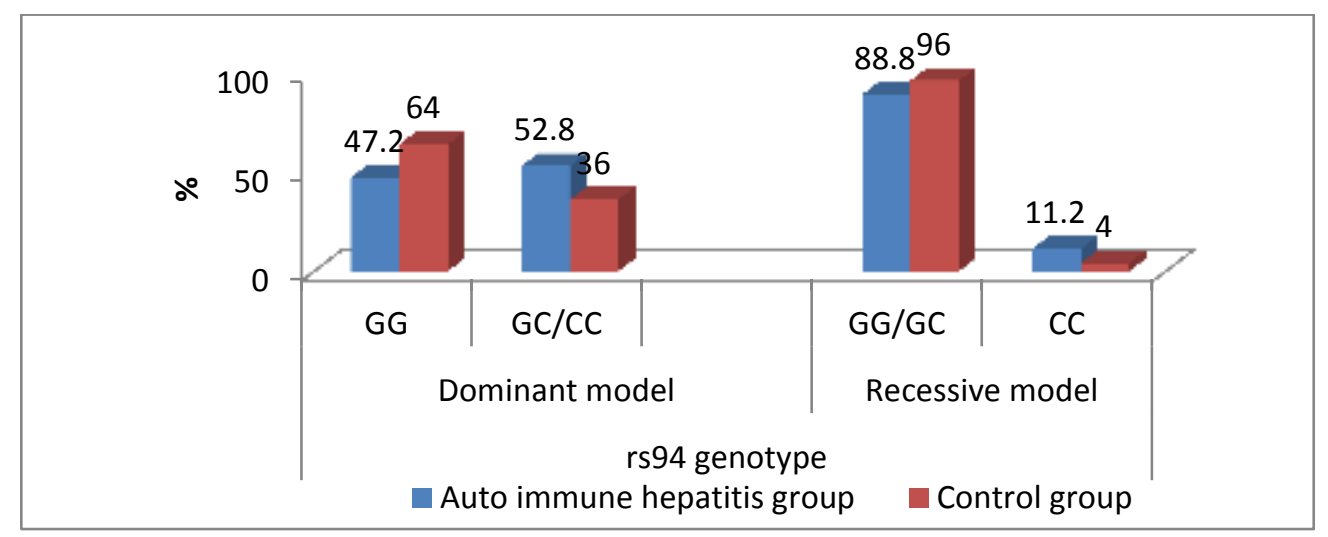

Fig. (5) Distribution of STAT 4 (RS7582694) dominant and recessive models among the studied group

Table (3) Comparison between STAT4 (rs7574865) and socio-demographic characters of autoimmune hepatitis patients.

\begin{tabular}{|c|c|c|c|c|c|c|c|}
\hline \multirow[t]{2}{*}{$\begin{array}{l}\text { rs65 } \\
\text { Variable }\end{array}$} & & \multicolumn{2}{|c|}{$\begin{array}{c}\text { Wild form } \\
\text { GG } \\
(\mathbf{n}=\mathbf{6 1})\end{array}$} & \multicolumn{2}{|c|}{$\begin{array}{c}\text { Mutant form } \\
\text { GT/TT } \\
(n=64)\end{array}$} & \multirow[t]{2}{*}{ Test of sig } & \multirow[t]{2}{*}{$\mathbf{P}$} \\
\hline & & No. & $\%$ & No. & $\%$ & & \\
\hline Sex & $\begin{array}{c}\text { Male } \\
\text { Female }\end{array}$ & $\begin{array}{l}30 \\
31\end{array}$ & $\begin{array}{l}49.2 \\
50.8\end{array}$ & $\begin{array}{l}27 \\
37\end{array}$ & $\begin{array}{l}42.2 \\
57.8\end{array}$ & $X^{2}=0.62$ & $0.43(\mathrm{NS})$ \\
\hline Age (years) & $\begin{array}{c}\text { Mean } \pm \text { SD } \\
\text { Range }\end{array}$ & \multicolumn{2}{|c|}{$\begin{array}{c}10.1 \pm 3.5 \\
4-16\end{array}$} & \multicolumn{2}{|c|}{$\begin{array}{c}9.7 \pm 4.0 \\
4-16\end{array}$} & $\begin{array}{c}\mathrm{ZMWU}= \\
0.51\end{array}$ & $0.61(\mathrm{NS})$ \\
\hline
\end{tabular}




\begin{tabular}{lcccccccc}
\hline Consanguinity & Positive & 13 & 21.3 & 13 & 20.3 & \multirow{2}{*}{$\mathrm{X}^{2}=0.019$} & 0.89 (NS) \\
& Negative & 48 & 78.7 & 51 & 79.7 & & & \\
Other AID & No & 49 & 80.3 & 56 & 87.5 & $X^{2}=1.19$ & 0.27 (NS) \\
AID in first degree & Yes & 12 & 19.7 & 8 & 12.5 & & \multirow{2}{*}{0.91 (NS) } \\
relative & No & 51 & 83.6 & 53 & 82.8 & $X^{2}=0.014$ & 0.9 \\
\hline
\end{tabular}

ZMWU=Z value of Mann Whitney U test $\quad$ X2 = chi square test. AID: autoimmune disease. NS: non-significant. HS: highly significant. S: significant.

Table 4 showed that there was no statistically difference between STAT4 (rs7574865) gene group regarding weight, height, head circumference and body mass index, liver span and spleen span.
Table (5) shows that there is statistically nonsignificant difference among STAT4 (rs7574865) gene regarding IgG, AST, ALT, T. Bilirubin, D. Bilirubin, S. Protein, PT, PTT, INR, GGT, S. Creatinine, Auto antibodies (ANA, ASMA).

Table (4) Anthropometric measurements according to STAT4(rs7574865) among patients.

\begin{tabular}{|c|c|c|c|c|c|c|}
\hline \multirow[t]{2}{*}{ Variable } & \multicolumn{2}{|c|}{$\begin{array}{c}\text { Wild form } \\
\text { GG } \\
(n=61)\end{array}$} & \multicolumn{2}{|c|}{$\begin{array}{c}\text { Mutant form } \\
\text { GT/TT } \\
(n=64)\end{array}$} & \multirow[t]{2}{*}{$\begin{array}{c}\text { Student "t" } t \text { " } \\
\text { test }\end{array}$} & \multirow[t]{2}{*}{$\mathbf{P}$} \\
\hline & Mean & \pm SD & Mean & \pm SD & & \\
\hline Weight (kg) & 27.9 & 10.3 & 27.7 & 10.7 & 0.101 & $0.92(\mathrm{NS})$ \\
\hline Weight centile & 15.3 & 9.2 & 15.7 & 11.58 & $* 0.203$ & $0.84(\mathrm{NS})$ \\
\hline Height (cm) & 128.9 & 20.25 & 127.0 & 22.55 & 0.49 & $0.62(\mathrm{NS})$ \\
\hline Height centile & 19.3 & 12.79 & 21.9 & 14.94 & $* 1.12$ & $0.26(\mathrm{NS})$ \\
\hline HC (cm) & 50.4 & 2.05 & 50.3 & 2.28 & 0.22 & $0.82(\mathrm{NS})$ \\
\hline HC centile & 49.2 & 19.47 & 49.7 & 19.73 & $* 0.092$ & 0.93 (NS) \\
\hline BMI $\left(\mathrm{kg} / \mathrm{m}^{2}\right)$ & 15.5 & 1.82 & 15.9 & 2.10 & 1.03 & 0.304 (NS) \\
\hline BMI centile & 22.2 & 18.26 & 23.7 & 18.22 & $* 0.56$ & 0.58 (NS) \\
\hline Liver span & 14.9 & 2.60 & 14.8 & 2.4 & 0.36 & $0.72(\mathrm{NS})$ \\
\hline Spleen span & 12.9 & 2.87 & 12.7 & 3.27 & 0.295 & 0.77 (NS) \\
\hline
\end{tabular}

*ZMWU was used. NS: non-significant. HS: highly significant. S: significant. HC: head circumference. BMI: body mass index. $\mathrm{Cm}$ : centimeter. $\mathrm{Kg}$ : kilogram.

Table (5) Comparison between STAT4 (rs7574865) genotype and some laboratory parameters in autoimmune hepatitis Patients.

\begin{tabular}{|c|c|c|c|c|c|c|c|c|c|}
\hline \multicolumn{2}{|c|}{ Variable } & \multicolumn{3}{|c|}{$\begin{array}{c}\text { Wild form } \\
\text { GG } \\
(\mathbf{n}=61) \\
\end{array}$} & \multicolumn{3}{|c|}{$\begin{array}{c}\text { Mutant form } \\
\text { GT/TT } \\
(n=64)\end{array}$} & $\mathrm{Z}_{\mathrm{MWU}}$ test & $\mathbf{P}$ \\
\hline \multirow{2}{*}{\multicolumn{2}{|c|}{$\overline{\text { AST }}$}} & & & & & & & & 877 (NC) \\
\hline & & 245.2 & \pm & 164.0 & 224.1 & \pm & 138.7 & 0.28 & 0.77 (NS) \\
\hline \multicolumn{2}{|l|}{ ALT } & 217.9 & \pm & 141.7 & 207.1 & \pm & 132.4 & 0.54 & 0.58 (NS) \\
\hline \multicolumn{2}{|c|}{ T. bilirubin } & 3.02 & \pm & 0.60 & 2.95 & \pm & 0.65 & 0.62 & $0.53(\mathrm{NS})$ \\
\hline \multicolumn{2}{|c|}{ D. bilirubin } & 1.02 & \pm & 0.32 & 1.02 & \pm & 0.35 & 0.30 & $0.76(\mathrm{NS})$ \\
\hline \multicolumn{2}{|c|}{ S. albumin } & 3.23 & \pm & 0.58 & 3.31 & \pm & 0.57 & 0.41 & 0.67 (NS) \\
\hline \multicolumn{2}{|c|}{ S. protein } & 8.85 & \pm & 1.82 & 9.05 & \pm & 1.57 & 0.44 & 0.66 (NS) \\
\hline \multicolumn{2}{|l|}{ IgG } & 3415.6 & \pm & 1852.8 & 4054.8 & \pm & 1994.3 & 1.91 & $0.056(\mathrm{NS})$ \\
\hline \multicolumn{2}{|l|}{ PT } & 16.5 & \pm & 3.38 & 15.9 & \pm & 2.70 & 0.63 & $0.52(\mathrm{NS})$ \\
\hline \multicolumn{2}{|l|}{ PTT } & 43.0 & \pm & 6.95 & 40.7 & \pm & 7.31 & 1.66 & 0.095 (NS) \\
\hline \multicolumn{2}{|l|}{ INR } & 1.65 & \pm & 0.44 & 1.57 & \pm & 0.36 & 1.02 & $0.31(\mathrm{NS})$ \\
\hline \multicolumn{2}{|l|}{ GGT } & 57.8 & \pm & 16.3 & 55.6 & \pm & 19.65 & 0.81 & 0.41 (NS) \\
\hline \multicolumn{2}{|l|}{$\mathbf{A L P}$} & 231.8 & \pm & 146.8 & 220.1 & \pm & 126.57 & 0.29 & 0.76 (NS) \\
\hline \multicolumn{2}{|c|}{ creatanine } & 0.52 & \pm & 0.20 & 0.57 & \pm & 0.225 & 1.75 & 0.08 (NS) \\
\hline \multirow{2}{*}{ ANA } & positive & 50 & & & 50 & & & \multirow{2}{*}{0.29} & \multirow{2}{*}{$0.59(\mathrm{NS})$} \\
\hline & negative & 11 & & & 14 & & & & \\
\hline ASMA2 & $\begin{array}{l}\text { positive } \\
\text { negative }\end{array}$ & $\begin{array}{l}47 \\
14\end{array}$ & & & $\begin{array}{l}53 \\
11\end{array}$ & & & 0.65 & $0.42(\mathrm{NS})$ \\
\hline
\end{tabular}

NS: non-significant. HS: highly significant. S: significant. PT: prothrombin time. PTT: partial thrombin time. INR: international normalized ratio. ANA: antinuclear antibodies. ASMA: anti-smooth 
muscle antibody. AMA: antimitochondrial antibody. Anti LKM: anti-liver kidney microsomal antibody. IgG: total immunoglobulin levels. GGT:.......... ALT: alanine aminotransferase. AST: aspartate aminotransferase. there are non-significant differences between STAT4 (rs7574865) genotype and some diagnostic scores (MELD, PELD, CHILD p score,
RODC and SDC) scores. Also, there was no statistically difference between those with (rs7574865) gene in the studied group regarding response to treatment. There was no statistically difference between STAT4(rs7582694) gene group regarding weight, height, head circumference and body mass index, liver span and spleen span.

Table (6) Anthropometric measurements according to STAT4(rs7582694) among patients.

\begin{tabular}{|c|c|c|c|c|c|c|}
\hline \multirow[t]{2}{*}{ Variable } & \multicolumn{2}{|c|}{$\begin{array}{c}\text { Wild form } \\
\text { GG } \\
(\mathbf{n}=95) \\
\end{array}$} & \multicolumn{2}{|c|}{$\begin{array}{c}\text { Mutant form } \\
\text { GC/CC } \\
(n=66)\end{array}$} & \multirow[t]{2}{*}{$\begin{array}{c}\text { Student "} t \text { " } \\
\text { test }\end{array}$} & \multirow[t]{2}{*}{$\mathbf{P}$} \\
\hline & Mean & \pm SD & Mean & \pm SD & & \\
\hline Weight (kg) & 27.7 & 10.1 & 27.8 & 10.9 & 0.023 & $0.98(\mathrm{NS})$ \\
\hline Weight centile & 15.4 & 9.3 & 15.7 & 11.4 & $* 0.14$ & $0.88(\mathrm{NS})$ \\
\hline Height (cm) & 128.9 & 20.2 & 127.1 & 22.5 & 0.48 & $0.63(\mathrm{NS})$ \\
\hline Height centile & 19.7 & 12.8 & 21.5 & 14.9 & $* 0.67$ & $0.51(\mathrm{NS})$ \\
\hline HC (cm) & 50.5 & 2.08 & 50.3 & 2.25 & 0.29 & 0.77 (NS) \\
\hline HC centile & 49.1 & 19.5 & 49.8 & 19.6 & $* 0.18$ & $0.86(\mathrm{NS})$ \\
\hline BMI $\left(\mathrm{kg} / \mathrm{m}^{2}\right)$ & 15.6 & 1.83 & 15.9 & 2.08 & 1.13 & $0.26(\mathrm{NS})$ \\
\hline BMI centile & 22.7 & 18.4 & 23.3 & 18.1 & $* 0.22$ & $0.82(\mathrm{NS})$ \\
\hline Liver span & 15 & 2.60 & 14.8 & 2.4 & 0.21 & $0.83(\mathrm{NS})$ \\
\hline Spleen span & 13 & 2.86 & 12.7 & 3.26 & 0.39 & $0.69(\mathrm{NS})$ \\
\hline
\end{tabular}

*ZMWU was used. NS: non-significant. HS: highly significant. S: significant. HC: head circumference. BMI: body mass index. Cm: centimeter. Kg: kilogram.

\section{DISCUSSION}

In current study, there was statistically significant higher incidence of autoimmune disease and autoimmune disease in first degree relative in autoimmune hepatitis group. These results were in agreement with Porta et al., (2019) found among 828 children and adolescents with autoimmune hepatitis, $89.6 \%$ had autoimmune hepatitis- 1 and family history of autoimmune disease, including diabetes, thyroid disease, Behçet's disease, psoriasis, and vitiligo, was observed in $161(21.9 \%)$ patients with AIH-1. Also, in agreement with our study, Jiménez-Rivera et al., (2015) reported the incidence of pediatric AIH in Canada. Patient history of previous or concomitant autoimmune diseases was positive in 32\% (49 of 154) and included the following: ulcerative colitis, $12 \%(\mathrm{n}=18)$; Crohn's disease, $5 \%(n=8)$; rheumatoid arthritis, $4 \%(n=6)$; thyroid disease, $4 \%(n=6)$; and celiac disease, $2 \%(n=$ 3 ). Family history of autoimmune disease in firstdegree relatives was reported in $25 \%$ (38 of 154). [14]

In current study, there was statistically significant lower weight on centile, BMI on centile and the height on centile in auto immune hepatitis group than control group. Our results were in agreement with cases report by Alqahtani et al., (2017) presented 5 years old Saudi boy presented with history of jaundice, fever and dark urine for 5 days with previous similar history at the age of 3 years. Examination revealed that, the patient's weight was on the 5th percentile, height was on 25th percentile and head circumference was on 25th percentile. [15]

In current study, there were statistically significant higher ALT, AST, ALP, serum bilirubin (total, direct), immunoglobulin $\mathrm{G}$ ) and the bleeding profile (PT, PTT, INR) in autoimmune hepatitis group, while serum albumin and proteins were significantly lower in autoimmune hepatitis group. Similarly, in study by Pathak and Kamat, (2018) showed that, elevated levels of aspartate and alanine aminotransferases are usually the first laboratory abnormalities that are observed in AIH. [16] In line with our results, Behairy, (2017) found that, at the time of presentation, all the patients had increased levels of AST (1.4-24 folds), ALT (0.4-20 folds) above upper limits of normal. But in contrast, they found that, serum alkaline phosphatase, GGT levels, and serum albumin were normal.[17] Similarly, Jiménez-Rivera et al., (2015) showed serum albumin levels were below normal limits in $43 \%$ (62 of 144). An increased international normalized ratio was reported in $45 \%$ (67 of 148) among AIH cases. [14]

In current study, $\mathrm{G}$ allele is protective but $\mathrm{T}$ (rs7574865) \& C allele (rs7582694) are independent risk factors for AIH. Individuals with $\mathrm{T}$ allele significantly increased risk of AIH by 2.5 folds than those with $\mathrm{G}$ allele and $\mathrm{C}$ allele significantly increased risk of AIH by 1.88 folds than those with G allele. GT genotype (rs7574865) significantly increased the AIH risk by two folds and TT genotype by four folds than GG individuals. GC genotype significantly increased the AIH risk by 1.76 folds and CC genotype by 3.8 folds than GG individuals. When we analyzing the dominant model of inheritance of the minor allele $\mathrm{T}$, GT+TT genotypes were significantly associated with AIH patients than healthy control with about 2.5-fold increased risk of AIH. While by the recessive model, TT genotype was significantly associated with AIH patients than healthy control with 3 folds increased risk of AIH. While regarding the dominant model of inheritance of the minor allele $\mathrm{C}, \mathrm{GC}+\mathrm{CC}$ genotypes 
(rs7582694) were significantly associated with AIH patients than healthy control with about 2-fold increased risk. On the other hands, by the recessive model, CC genotype was significantly associated with AIH patients than healthy control with 3.03 -fold increased risk. Similarly, in study by Li et al., (2017) found all genotypes are distributed according to HWE in controls (all $\mathrm{p}$ values $>0.05$ ). The frequencies for rs7574865 $\mathrm{T}$ allele and rs7582694 $\mathrm{C}$ allele were significantly higher in type 1 AIH cases than control group $(30.6 \%$ vs $19.3 \%, 32.5 \%$ vs $20.0 \%)$, and the frequencies for rs2476601- $\mathrm{T}$ allele was significantly lower in in type 1 AIH cases than control group $(19.7 \%$ vs $30.0 \%)$. Logistic regression analysis showed that type $1 \mathrm{AIH}$ was significantly higher in carriers of T allele of rs7574865 than those with GG genotype (GT + TT versus $\mathrm{GG}$ ), and higher in carriers of $\mathrm{C}$ allele of rs7582694 than those with GG genotype (GC + CC versus GG). In addition, found type $1 \mathrm{AIH}$ risk was significantly lower in carriers of T allele of rs2476601 than those with $\mathrm{CC}$ genotype (CT $+\mathrm{TT}$ versus $\mathrm{CC}) . \mathrm{Li}$ et al., found that participants with GC or CC of rs7582694 and $\mathrm{CC}$ of rs2476601 genotype have the highest type 1 AIH risk, compared to participants with GG of rs7582694 and CT or TT of rs2476601 genotype, after covariates adjustment. [18]

In addition, meta-analysis by Liang et al., (2012) demonstrated that the STAT4 rs7574865 $\mathrm{T}$ allele conferred susceptibility to various autoimmune diseases, suggesting an association between STAT4 gene polymorphism and autoimmune diseases [19]

In disagreement with current study suggested that, $\mathrm{G}$ allele had protective effect, Jiang et al., (2013) identify genetic susceptibility loci for hepatitis B virus (HBV)-related hepatocellular carcinoma (HCC) in the Chinese population, we carried out a genome-wide association study (GWAS) in 2,514 chronic HBV carriers (1,161 HCC cases and 1,353 controls) followed by a 2-stage validation among 6 independent populations of chronic HBV carriers $(4,319$ cases and 4,966 controls). they showed that $G$ allele at rs7574865 was associated with increased risk for HCC, suggesting dual roles of STAT4 in autoimmune diseases and HBV-related HCC. Interestingly, subjects with GG genotype at rs7574865 had the lowest mRNA levels of STAT4 in both HCC and non-tumor tissues compared with TG and TT genotypes. The differences observed between our results and previous studies could be due to diversity in susceptibility genes between the different ethnic populations (allele $\mathrm{T}$ is observed with a frequency of around 35\% in East Asian populations from 1000 Genomes) and the antigens presented to lymphocytes leading to autoimmune responses directed against hepatocytes. [20]

We found both dominant and recessive models of STAT4 (rs7574865) are significant predictors of autoimmune hepatitis (dominant model was 5.63 times more risk and the recessive model was 2.77 times more risk). While regarding STAT4 (rs7582694) only the dominant model was significant predictor (3.1 times more than normal). STAT4 is involved in the activation of dendritic cells and macrophages, as well as signal transduction within activated peripheral blood monocytes, while PTPN22, which is found in the cytosol of immune cells, is involved in many immune signalling pathways, including lymphocyte activation and proliferation. The STAT4 and PTPN22 genes have previously been linked to the onset of AIH and autoimmune disorders including rheumatoid arthritis (RA) and systemic lupus erythematosus (SLE). TGF is a cytokine that plays an important role in immune response regulation. Two functional polymorphisms in the TGF1 gene's coding region (codon 10 and codon 25) have been linked to increased TGF $\beta 1$ expression levels and severe progression of AIH[21] The STAT4 transcription factor would be an effective genetic factor inducing the development of IL-22, which would play a pathological function in autoimmune hepatitis by inducing the production of IL-17 and effector cell differentiation [22].

These results similar to Abelson et al., (2009) confirm and define the genetic association of STAT4 and systemic lupus erythematosus (SLE), investigate the possibility of correlations with differential splicing and/or expression levels, and genetic interaction with IRF5, in their study, 30 tag SNPs were genotyped in an independent set of Spanish cases and controls. SNPs surviving correction for multiple tests were genotyped in five new sets of cases and controls for replication. STAT4 cDNA was analysed by 5 '-RACE PCR and sequencing. Expression levels were measured by quantitative PCR. They showed a significant association of STAT4 polymorphism with predisposition to $\mathrm{AIH}(\mathrm{OR}=1.61$, $\mathrm{P}=0.001)$ especially in the Japanese and Chinese populations [23]

In agreement with current results, Alvarez et al., (1999) suggested that STAT4 polymorphism was positively associated type-1 AIH risk. STAT4 was one type of critical transcription factor involved in the Th1/Th2 cytokine balance regulation. [24] Moreover, De Boer et al., (2014) used a genome-wide approach to identify genetic variants that predispose individuals to AIH. They performed a genome-wide association study of 649 adults in the Netherlands with AIH type 1 and 13,436 controls. Initial associations were further analyzed in an independent replication panel comprising 451 patients with AIH type 1 in Germany and 4103 controls. Their study indicated a strong association between the genes encoding the immunoregulatory molecules such as the transcription factor STAT4, with susceptibility to autoimmune hepatitis. [25]

In current study, there was statistically nonsignificant relation between STAT4 (rs7574865) \& (rs7582694) gene and age, sex, parent's consanguinity, other autoimmune disease and autoimmune disease in first degree relatives among the studied group. This was in line with our study, Nageeb et al., (2018) evaluate the association of STAT4 gene polymorphism with multiple sclerosis (MS) and juvenile onset systemic lupus erythematosus (JO-SLE) and its relation to disease severity and included group 1 consisted of 40 multiple sclerosis (MS) patients while group 2 
included 40 juvenile onset systemic lupus erythematosus (JO-SLE) patients and forty healthy volunteers (controls). They showed that there was no significant difference between clinical parameters (age, sex, disease duration, course and number of attack) of MS patients and STAT4 CC (rs7582694) polymorphic genotype [26]

In current study, there was statistically nonsignificant difference among STAT4 (rs7574865) \& (rs7582694) gene group (Wild form GG \& Mutant form GT/TT) regarding AST, ALT, T. Bilirubin, D. Bilirubin, S. Protein, PT, PTT, INR, GGT, S. creatinine, auto antibodies (ANA, ASMA). while there was statistically significant difference among STAT4 (rs7582694) gene group regarding IgG. In line with current results El Bassuonia et al., (2016) investigated the prevalence of the STAT4 G > C (rs7582694) polymorphism in patients with systemic lupus erythematosus (SLE) and controls in a sample of the Egyptian population. They reported that in their study, the distribution of the STAT4 (rs7582694) polymorphism among the SLE patients in terms of different symptoms and the anti-dsDNA titer showed an insignificant difference ( $P>0.05$ ). Comparison of the distribution of polymorphisms among the patients in terms of hematological parameters (HB, platelet and WBC), an insignificant difference between different types of polymorphisms, Comparison of the distribution of polymorphism among the patients in terms of renal function, with an insignificant difference between different types of polymorphisms, Also, Taylor et al., (2011) studied 22 SLE susceptibility polymorphisms with previous genome-wide evidence of association $(\mathrm{p}<5 \times 10-8)$ in 1919 SLE cases from 9 independent Caucasian SLE case series and 4813 independent controls. they agreed that for SLE patients in rs7574865 correlated with proteinuria, and antidsDNA positivity.

Moreover, Izadkhasti et al., (2018) study, the prevalence of STAT4 gene G/C (rs7582694) single nucleotide polymorphism (SNP) in the patients with SLE $(n=122)$ and in control group $(n=127)$ was investigated among a sample population from Lorestan province they found that, the prevalence of STAT4 gene (rs7582694) in the patients with SLE and in control group was investigated, and they found no relationship between these polymorphisms and ACR criteria for SLE. Also, no significant relationship between anti-dsDNA and ANA with the type of the genotype. In contrast, in Nageeb et al., (2018) study included group 1 consisted of 40 Multiple sclerosis (MS) patients while group 2 included 40 juvenile onset systemic lupus erythematosus (JO-SLE) patients and forty healthy volunteers (controls). They found that, the STAT4 CC (rs7582694) polymorphic genotype associated with increased ANA positivity, anti-dsDNA, anti Sm antibodies'

In current study, there are non-significant differences between STAT4 (rs7574865) \& (rs7582694) genotype group (Wild form GG \& Mutant form GT/TT) and diagnostic scores (MELD, PELD, Child B score, RODC and SDC) scores and regarding response to treatment. Similar results were reported by
Nageeb et al., (2018) found no significant difference was detected in Kurtzke's Expanded Disability Status Scale (EDSS) comparing different STATE 4 (rs7582694) polymorphic genotypes. STAT4 CC genotype showed a significant increase in the SLE activity index (SLEAI) score and damage index as compared to the STAT4 GG genotype patients. [26] This is in discordance with Taylor et al. (2011) reported the significant association of STAT4 polymorphism with severe renal insufficiency. [27]

\section{Conclusion}

The results of current study indicated that rs7574865 and rs7582694 in STAT4 gene minor alleles are associated with increased type 1 AIH risk.

\section{References}

[1] A.J.Czaja, Transitioning from idiopathic to explainable autoimmune hepatitis. Digestive diseases and sciences. vol 60(10), pp. 2881-900, 2015.

[2] A.J.Czaja,M.P.Manns, Advances in the diagnosis, pathogenesis, and management of autoimmune hepatitis. Gastroenterology. vol 139(1),e4, pp. 5872,2010 .

[3] N.Kaur,R.W.Minz,S.Anand,B.Saikia,R. Aggarwal ,A.Das, et al. HLA DRB1 alleles discriminate the manifestation of autoimmune hepatitis as type 1 or type 2 in North Indian population. Journal of clinical and experimental hepatology. vol 4(1), pp. $14-8,2014$

[4] M.S.Longhi ,Y. Ma ,G. Mieli-Vergani,D. Vergani,Aetiopathogenesis of autoimmune hepatitis. Journal of autoimmunity. vol 34(1), pp. 7-14, 2010.

[5] S. E. Baranzini, The genetics of autoimmune diseases: a networked perspective. Current opinion in immunology. vol 21(6), pp. 596-605, 2009.

[6] N.Yan , S. Meng , J. Zhou ,J. Xu ,F. S. Muhali , W. Jiang,et al, Association between STAT4 gene polymorphisms and autoimmune thyroid diseases in a Chinese population. International journal of molecular sciences. vol 15(7), pp. 1228012293,2014.

[7] M.H.Kaplan,ACritical Regulator of Inflammation In Vivo. Immunologic Research. vol 32(1), pp. 231-241,2005.

[8] W. T. Watford , B. D. Hissong ,J. H. Bream , Y. Kanno ,L. Muul , J. J. O'Shea, Signaling by IL-12 and IL-23 and the immunoregulatory roles of STAT4. Immunological reviews. vol 202(1), p 139-156,2004.

[9] E. Bettelli ,T. Korn ,V. K. Kuchroo, Th17: the third member of the effector $\mathrm{T}$ cell trilogy. Current opinion in immunology. vol 19(6), pp.652-657,2007.

[10] E. M. Hennes ,M. Zeniya ,A. J. Czaja ,A. Parés , G. N. Dalekos ,E. L. Krawitt, et al. Simplified criteria for the diagnosis of autoimmune hepatitis. Hepatology. vol 48(1), pp. 169-176,2008. 
[11] W.W.Wilfinger,K.Mackey,P.Chomczynski, Effect of $\mathrm{pH}$ and ionic strength on the spectrophotometric assessment of nucleic acid purity. Biotechniques. vol 22(3), pp. 474481,1997.

[12] H. M. El-Saadany , W. H. Amer ,H. S. Khalil, R. A. Gaber, S.A.Elshweikh, Association of STAT4 polymorphism with susceptibility and severity of rheumatoid arthritis and systemic lupus erythematosus in Egyptian patients. The Egyptian Rheumatologist. vol 38(1),pp. 2127,2016.

[13] S.Rodriguez,T.R.Gaunt,I.N.Day, HardyWeinberg equilibrium testing of biological ascertainment for Mendelian randomization studies. American journal of epidemiology. vol 169(4), pp. 505-514,2009.

[14] C.Jiménez-Rivera ,S. C. Ling, N. Ahmed, J. Yap ,M. Aglipay ,N. Barrowman, et al. Incidence and characteristics of autoimmune hepatitis. Pediatrics. vol 136(5), pp.1237-1248,2015.

[15] Y. A. Alqahtani ,S. M. Alqahtani ,J. A. Alfaifi , A. A. Alghamdi ,O. M. Alrufaidy, Autoimmune hepatitis in children: a case report. The Egyptian Journal of Hospital Medicine. vol 66(1),pp. 152155,2017.

[16] S. Pathak ,D. Kamat . Autoimmune hepatitis in children. Pediatric annals. vol 47(2), pp.8186,2018 .

[17] O. G. A.Behairy, Characteristics of autoimmune hepatitis in a sample of Egyptian children. Egyptian Pediatric Association Gazette. vol 65(4), pp.108-113,2017.

[18] X. Li ,H. Chen,Y.Cai,P. Zhang ,Z. Chen,Association of STAT4 and PTPN22 polymorphisms and their interactions with type-1 autoimmune hepatitis susceptibility in Chinese Han children. Oncotarget. vol8(37),pp.60933,2017.

[19] Y.L.Liang ,H. Wu ,X. Shen ,P. Q. Li ,X. Q. Yang, L. Liang, et al. Association of STAT4 rs7574865 polymorphism with autoimmune diseases: a meta-analysis. Molecular biology reports. vol39(9),pp.8873-8882,2012.

[20] D. K. Jiang D,J. Sun ,G. Cao ,Y. Liu ,D. Lin ,Y. Z. Gao, et al. Genetic variants in STAT4 and
HLA-DQ genes confer risk of hepatitis B virusrelated hepatocellular carcinoma. Nature genetics. vol45(1),pp.72-75,2013.

[21] O. B. Ramírez ,J. M. Rincón, R. B. Cobos , I. A. Ávila ,J. R. Bello, STAT4 confers risk for rheumatoid arthritis and systemic lupus erythematosus in Mexican patients. Immunology letters. vol175,pp.40-43,2016.

[22] E.F.Remmers,R.M.Plenge,A.T.Lee,R.R. Graham,G. Hom,T. W. Behrens, et al. STAT4 and the risk of rheumatoid arthritis and systemic lupus erythematosus. New England Journal of Medicine. vol357(10),pp. 977-986,2007.

[23] A.K.Abelson,A.M.DelgadoVega,S.V.Kozyrev,E.Sanchez,R.VelazquezCruz,N.Eriksson,et al. STAT4 associates with systemic lupus erythematosus through two independent effects that correlate with gene expression and act additively with IRF5 to increase risk. Annals of the rheumatic diseases. vol68(11),pp.1746-1753,2009.

[24] F. Alvarez ,P. Berg,F. B. Bianchi ,L. Bianchi, A. Burroughs ,E. L. Cancado, et al. International Autoimmune Hepatitis Group Report: review of criteria for diagnosis of autoimmune hepatitis. Journal of hepatology. vol31(5),pp.929-938,1999.

[25] Y. S. De Boer ,N. M. van Gerven,A. Zwiers , B. J. Verwer, B. van Hoek ,K. J. van Erpecum , et al. Genome-wide association study identifies variants associated with autoimmune hepatitis type 1. Gastroenterology. vol147(2)e5, pp.443452,2014

[26] R. S. Nageeb ,A. A. Omran ,G. S. Nageeb , M. A. Yousef ,Y. A. A. Mohammad ,A. Fawzy, STAT4 gene polymorphism in two major autoimmune diseases (multiple sclerosis and juvenile onset systemic lupus erythematosus) and its relation to disease severity. The Egyptian journal of neurology, psychiatry and neurosurgery. vol54(1), pp.16, 2018.

[27] K.E.Taylor,S.A.Chung,R.R.Graham,W.A.Ort mann ,A. T. Lee ,C. D. Langefeld, et al. Risk alleles for systemic lupus erythematosus in a large case-control collection and associations with clinical subphenotypes. PLoS genetics. vol 7(2), 2011. 